\title{
Wissen im Entwurf
}

Benjamin Meyer-Krahmer

Christoph Hoffmann (Hg.) 2008: Daten sichern. Schreiben und Zeichnen als Verfahren der Aufzeichnung [=Wissen im Entwurf, 1], Zürich: diaphanes, brosch., 208 S., 24,90€, ISBN-13: 978-3-03734-048-6.

Barbara Wittmann (Hg.) 2009: Spuren erzeugen. Zeichnen und Schreiben als Verfahren der Selbstaufzeichnung [=Wissen im Entwurf, 2], Zürich: diaphanes, brosch., 200 S., 24,90€, ISBN-13: 978-303734-070-7.

Karin Krauthausen und Omar W. Nasim (Hg.) 2010: Notieren, Skizzieren. Schreiben und Zeichnen als Verfahren des Entwurfs [=Wissen im Entwurf, 3], Zürich: diaphanes, brosch., 224 S., 24,90 €, ISBN-13: 978-3-03734-053-0.

Jutta Voorhoeve (Hg.) 2011: Welten schaffen. Zeichnen und Schreiben als Verfahren der Konstruktion [=Wissen im Entwurf, 4], Zürich: diaphanes, brosch., 200 S., 24,90€, ISBN-13: 978-3-03734-054-7.

Nicht nur inhaltlich, auch konzeptuell macht die vierbändige Reihe Wissen im Entwurf ihrem Titel alle Ehre: Die "heuristische Suspendierung“ (Bd. 1: 8) einer ganzen Palette von gewöhnlich sorgfältig aufrechterhaltenen Unterscheidungen (zwischen Disziplinen, Wissensgebieten, Künsten und Wissenschaften, Epochen und so weiter) steht am Anfang dieses Unterfangens und eröffnet so die Möglichkeit einer vielstimmigen Untersuchung von "Schreiben und Zeichnen als Verfahren" in Wissenschaften und Künsten, genauer: „dem je spezifischen Zusammenspiel von Hand, Stift und Papier in epistemischen und ästhetischen Verfahren" (Klappentext). Entsprechend der heuristischen Haltung, die sich unter anderem in der interdisziplinären Anlage der Publikation manifestiert, formuliert Hans-Jörg Rheinberger in einem der 
Beiträge programmatisch: „Wissenschaft ist Entwurf, aber ein Entwurf, bei dem man nicht genau sieht, wohin der Wurf geht." (3: 143)

Damit ist auch eine große Stärke der Publikation benannt, deren experimenteller Charakter sich in einer Konstellation von 30 Beiträgen ausdrückt, die jeweils für sich genommen lesenswert und erhellend, zugleich jedoch Teil eines Projekts („Wurfs“) sind, dessen Ausgang in der Theorie nicht zu antizipieren ist. Tatsächlich verdichtet sich während der abwechslungsreichen Lektüre der Eindruck, dass es wie in den von den Autoren und Autorinnen beschriebenen Versuchs-, Schreibe- und Zeichenanordnungen notwendig ist, das Experiment dieser Publikation durchzuführen, um herausfinden zu können, welche Erkenntnisse mittels dieses Ansatzes und seiner Methode zu gewinnen sind und wo deren Grenzen liegen. Dies sollte eigentlich bei jedem Sammelband der Fall sein, findet sich hier jedoch dank einer gelungenen Balance von solider Forschung und risikofreudigem wissenschaftlichen Geist besonders produktiv umgesetzt. Denn bei allem unbestreitbaren Gewinn scheint es eines gewissen editorischen wie konzeptionellen Wagemuts $\mathrm{zu}$ bedürfen, um beispielsweise unter dem Thema „Zeichnen und Schreiben als Verfahren der Selbstaufzeichnung“ neben Versuchsanordnungen zur Erforschung der Handschrift in der Psychiatrie um 1900 auch Zeichnungen des Künstlers Martin Kippenberger (1953-1997) zu verhandeln.

Bezüglich der Gesamtkonzeption haben die Einleitung von Christoph Hoffmann im ersten Band, auf die sich mehrere Autoren in ihren Beiträgen beziehen, sowie ein Interview mit Hans-Jörg Rheinberger zu „Papierpraktiken im Labor" im dritten Band einen besonderen Stellenwert, da sie einige der tragenden programmatischen Annahmen explizieren. Hoffmann erläutert den für alle Bände zentralen Begriff des „Verfahrens“ wie folgt: „Im Schreiben und Zeichnen werden nicht nur Wissensbestände bewahrt und übermittelt. Es ergeben sich zugleich spezifische Möglichkeiten, Erfahrungen und Überlegungen neu anzuordnen. Schreiben und Zeichnen müssen auch als epistemische Verfahren verstanden werden, die im Akt der Aufzeichnung an der Entfaltung von Gegenständen des Wissens teilhaben." (1: 7)

Das Augenmerk wird also sowohl auf den epistemisch relevanten Schritt der Veräußerlichung („Aufzeichnung“) als auch auf den der Weiterverarbeitung („Neuanordnung“") des Aufgezeichneten gerichtet. Hoffmann stellt weiter fest, dass entsprechend der Einsicht, dass „die Gegenstände der Wissenschaft von den Verfahren ihrer Darstellung nicht ohne weiteres abzutrennen sind“, technisch komplizierte „Repräsentationsvorgänge“ (1:7) in der Wissenschaftsforschung schon länger studiert, demgegenüber jedoch die technisch simplen Verfahren des Schreibens und Zeichnens vernachlässigt wurden. Dabei bleiben selbst unter aktuellen hightech Bedingungen heutiger Forschungseinrichtungen lowtech Papiertechniken relevant: „Hier, im Abzeichnen, Umreißen, Ab- und Aufschreiben, wird fixiert und probeweise $\mathrm{zu}$ den vorausgehenden Überlegungen in Bezug gesetzt, was sich fürs Erste 
ergeben hat und im Weiteren zu verstehen ist. Schreiben und Zeichnen bilden die Mittel eines Wissens im Entwurf." (1: 8)

Konstituierend wirken Zeichnen und Schreiben beim Entwerfen von Wissen in jedem Stadium eines solchen Prozesses jedoch nicht nur entfaltend, sondern auch einschränkend mit, indem sich nämlich „[i]m Aufzeichnen entscheidet, was als Aufgezeichnetes schließlich fortwirken kann" (1: 10). So wird im Aufzeichnen der weitere Verlauf eines Experiments oder der Arbeit an einem philosophischen Problem stets vorgeprägt, wenn nicht gar vorgeschrieben. Eine weitere konstituierende Dimension der hier untersuchten Verfahren liegt im präfigurierenden Effekt einmal erstellter bildlicher Darstellungen auf jede nachfolgende Beobachtung und damit eventuell einhergehende, neu erstellte oder überarbeitete Darstellungen. Da diese prospektiven Effekte des Aufzeichnens nur bedingt plan- oder kontrollierbar sind, haben wir es hier mit Phänomenen zu tun, die auf die jedem Verfahren innewohnende Kontingenz verweisen.

Ausgehend von der Annahme, dass es sich beim Schreiben und Zeichnen im Rahmen des Aufzeichnens um "gerichtete, strukturierte Abläufe" handle, sieht Hoffmann das „enger Verfahrensmäßige“ in den „,je besonderen im Schreiben und Zeichnen realisierten Aktivitäten“. (1: 12) Als Beispiele hierfür werden das Anfertigen eines Diagramms, das Führen eines Protokolls, das Schreiben einer Notiz in den Marginalien eines Buches sowie das zeichnerische Erfassen eines Objekts am Mikroskop genannt (1: 13). Zu Perspektive und Methode der Untersuchung solcher graphischer Praktiken unter dem Aspekt des Verfahrens heißt es: „In der Durchsicht größerer Folgen von Aufzeichnungen kann man ikonische und symbolische Repertoires verzeichnen, Typologien des Vorgehens unterscheiden, Routinen identifizieren und so allmählich lernen, im Aufgezeichneten und in gewissem Maße gegen dessen Inhalt, der nach einer anderen Form des Studiums verlangt, das Prozedurale der Aufzeichnung zu erkennen." (Ebd.) Diese Verschiebung der Aufmerksamkeit „vom Produkt auf die Aktivität“ (ebd.) steht im Zentrum des Publikations- wie des Forschungsprojekts „Wissen im Entwurf“. Dass die Publikation ausschließlich Materialstudien enthält, wird ebenfalls mit dem Begriff des Verfahrens begründet, der ,auf eine situative Logik der Ausführung“ verweise - „Verfahrensweisen abseits ihrer Umsetzung zu erfassen, geht jedoch am Kern vorbei“. (1: 15) Diese Konzeption führt dazu, dass die Beiträge eine Tendenz aufweisen, primär quellenimmanent $\mathrm{zu}$ argumentieren und weniger auf den jeweiligen Forschungsstand einzugehen.

Das Übergewicht der Wissenschaften gegenüber den Künsten, das aus den hier wiedergegebenen Aussagen hervorgeht, ist repräsentativ für die Publikation insgesamt. Dennoch tragen die Bände zu einem besseren Verständnis von Zeichnen und Schreiben in beiden Bereichen bei, da die eingangs proklamierte Heuristik auch in diesem Sinne funktioniert: Die Fokussierung auf das Verfahrensmäßige in künstlerischen und wissenschaftlichen Praktiken 
erlaubt in vielen Fällen den - seinerseits heuristischen - Transfer über Disziplingrenzen hinweg.

Im Hinblick auf die Materialität der untersuchten Gegenstände handelt es sich um Inskriptionen simpler Schreibwerkzeuge auf Papier unterschiedlicher Art. Unter „Stift“ fällt nicht nur der Bleistift, der auf der Website des Projekts zum Paradigma des sich mit einfachsten Mitteln aufzeichnenden Geistes stilisiert ist, sondern auch die (Zeichen- und Schreib-) Feder, mit deren Hilfe Tinte oder Tusche aufgetragen werden, sowie Rötelstift, Buntstift, (Fett- und Pastell-) Kreide, Kohle und weitere, nicht näher bestimmte Instrumente zum ein- oder mehrfarbigen Schreiben und Zeichnen. Ebenso heterogen sind die unter „Papier“ gefassten Beschreibstoffe, zu denen linierte und leere Notizbücher, Zettel, lose Bögen von Zeichen- und Transparentpapier zählen, aber auch Formulare, denen vorgängiges Wissen eingeschrieben ist, das die Eintragungen reguliert.

Dank der hohen Abbildungsqualität, die Details der Inskription und deren Materialität wiedergibt, sowie der zahlreichen, teils ganzseitigen Farbabbildungen vermittelt die Publikation nicht nur auf der Text- sondern auch auf der Bildebene einen Eindruck von der Vielfalt und der ästhetischen Qualität der graphischen Artefakte. Die Abbildungen erfüllen in diesem Zusammenhang nicht die Funktion illustrierender Verweise, sondern sind Teil der Argumentation. Denn die abgebildeten Inskriptionen sind die Spuren der Interaktionen der Wissenschaftlerinnen und Wissenschaftler beziehungsweise der Künstlerinnen und Künstler mit der Welt, auf die es hier ankommt - entsprechend dem von Rheinberger wie folgt markierten Ausgangspunkt des gesamten Forschungs- und Publikationsunterfangens:

Im Ganzen betrachtet ist die Wissenschaft ein kulturelles Unternehmen mit einer eigenen Materialität. Es kann sein, dass die Materialität der wissenschaftlichen Praxis sich von anderen Feldern unterscheidet, in denen es ebenfalls um materiale Praktiken geht, wie zum Beispiel der bildenden Kunst. „Le réel scientifique“ hat, wie Gaston Bachelard schrieb, eine eigene Bedingtheit, und ich denke, die Wissenschaftsgeschichte sollte diese „Bedingtheit der wissenschaftliche[n] Wirklichkeit" untersuchen. Die Untersuchung der materialen Praktiken der Wissenschaften wendet sich also gegen eine durchaus verdienstvolle Form der Ideengeschichte, um die Erfahrung des Labors in die Wissenschaftsgeschichte zu integrieren. (3: 140)

Im Zentrum des Erkenntnisinteresses von Wissen im Entwurf stehen also das Prozedurale des epistemischen und ästhetischen Entwerfens sowie die je eigene Materialität, die sich abhängig vom untersuchten Bereich (zum Beispiel Wissenschaft oder Kunst) in den jeweiligen Praktiken finden lässt. Die Frage, inwiefern Ästhetik für Forschung und wissenschaftliche Erkenntnis über deren Abhängigkeit von Darstellungsverfahren hinaus relevant ist, wird ebenso wenig beantwortet wie die Frage, inwiefern das in den Künsten entworfene Wissen mit dem aus Forschungsprozessen hervorgehenden 
vergleichbar ist. Die Publikation stellt eine Fülle von Material zur Beschäftigung mit diesen Fragen bereit, überlässt deren Kontemplation gleichwohl dem Leser und der Leserin selbst. Was genau leistet also die Kompilation von Materialstudien, die die unterschiedlichen Formen von Schreiben und Zeichnen aus den unterschiedlichen Wissensgebieten zueinander in Beziehung setzt?

Zunächst einmal beweisen die Fülle und Heterogenität des in den Beiträgen untersuchten Materials, dass Schreiben und Zeichnen als Verfahren tatsächlich in diversen wissenschaftlichen und künstlerischen Praktiken zum Einsatz kommen. Daran anschließend lohnt sich zu fragen, inwiefern es sich bei diesen untersuchten Prozessen um solche handelt, die (primär) darauf ausgerichtet sind, Wissen zu generieren oder besser: welche Arten von Wissen hier angesprochen sind. ${ }^{1}$ Es erscheint hilfreich, an dieser Stelle eine Auflistung der Arbeitsvorgänge einzufügen, die mit Hilfe der Verfahren Zeichnen und Schreiben ausgeführt werden: ${ }^{2}$

- Band 1 Aufzeichnung: Katalogisierung stellarer Nebel in der Astronomie, Identifizierung eines Typusexemplars in der Zoologie, kunsthistorische Erfassung von Gemälden, Entwerfen einer philosophischen Abhandlung, Planen eines literarischen Textes, Dokumentation von Sektionen in der Pathologie.

- Band 2 Selbstaufzeichnung: Erforschung des Schreibvorgangs in der Psychiatrie um 1900, Erforschung der Handschrift um 1900, Traumdeutung, Psychoanalyse, ${ }^{3}$ Zeichnen von sich bewegenden Körpern, Selbstaufzeichnung.

- Band 3 Entwurf: Verfassen eines philosophischen Textes, Hervorbringen des Schreibens und Denkens, Notation topologischer Objekte, Aufzeichnen von Ergebnissen im Labor durchgeführter Ergebnisse, Erkennen und Erkunden stellarer Nebel, Zeichnen als Entwerfen und (Lebens-) Werk.

- Band 4 Konstruktion: Entwerfen von Schiffsrümpfen, Konstruieren von Brücken, Homöopathische Diagnostik, Konstruieren literarischer Texte, Reflexion der Perspektivzeichnung, zeichnerische Konstruktion mittels Hilfslinien, Computeranimation, architektonisches Entwerfen und Konstruieren.

In dieser stark verkürzenden Liste finden sich Punkte, die sich sowohl zu Verbindungs- als auch zu Trennungslinien zusammenfügen lassen. Beim zweiten Blick auf die Vielzahl der behandelten Projekte fällt auf, dass in dem den Beiträgen zugrundeliegenden Material Zeichnen und Schreiben nicht nur als Verfahren angesprochen werden, sondern auch als Gegenstand der Forschung sowie als Medium und Gegenstand primär selbstreflexiver Konstellationen. In diesem Sinne lässt sich das von den Autorinnen und Autoren untersuchte Material im Hinblick darauf, wie Zeichnen und Schreiben dort jeweils erscheinen, grob in drei Gruppen unterteilen: 
1. Zeichnen und Schreiben als Instrument wissenschaftlicher Erkenntnis und technischer Konstruktion (Astronomie, Zoologie, Biologie, Topologie, Brücken- und Schiffsbau)

2. Zeichnen und Schreiben als Gegenstand der Forschung (Graphologie, Schriftkunde, Psychoanalyse)

3. Zeichnen und Schreiben als Gegenstand des Zeichnens und Schreibens (literarisches Schreiben, künstlerisches Zeichnen).

Dies bedeutet zudem, dass die Akteure, die die untersuchten Inskriptionen hervorbringen, unterschiedliche Rollen erfüllen und demnach die Motivation $\mathrm{zu}$ zeichnen oder zu schreiben von ganz unterschiedlicher Art ist: Wissenschaftler und Künstler stehen so (beispielsweise in Band 2) anonymen Versuchspersonen und Patienten gegenüber. Dem um Systematisierung bemühten Leser stellt sich insofern ein Problem, als der unter Punkt 2 genannte Zugang zum Zeichnen und Schreiben nur schwer mit den die Publikation rahmenden Fragen in Verbindung zu bringen ist. Die Anlage der Publikation gerät hier an ihre Grenzen, denn es stehen nicht Zeichnen und Schreiben als Verfahren im Zentrum, sondern die Interpretation von Inskriptionen anderer durch Wissenschaftler und Psychoanalytiker. Im Falle der Untersuchung von Markus Klammers Beitrag verhält es sich so, dass der Patient schreibt und zeichnet und Freud dieses Material verwendet (2: 69107). Es geht also um Schreiben und Zeichnen als Darstellungsverfahren von Symptomen für einen interpretierenden Experten. Der Logik der Publikation folgend müsste jedoch eigentlich primär das Schreiben und Zeichnen Freuds analysiert werden. Dies ist nur insofern gegeben als Freuds strategische, editorische Weiterverarbeitung der Zeichnung und des Textes seines Patienten sowie deren argumentative Stellung in seiner Theorie der Psychoanalyse ein Thema des Beitrags ist.

Doch zurück zu den weit wichtigeren Verbindungslinien, die sich durch alle Bände der Publikation verfolgen lassen. Dass Zeichnen und Schreiben in den Naturwissenschaften eine prominente Rolle spielen, ist bekannt und an sich wenig überraschend. In welchem Maße diese Aktivitäten jedoch als Verfahren eine konstituierende Funktion im Hinblick auf den jeweiligen Untersuchungsgegenstand haben, lässt sich erst auf der Basis von Materialstudien nachvollziehen. Dies gelingt in der Reihe spielend durch die Qualität der Beiträge und deren konsequente Fokussierung auf die übergeordneten Themenstellungen der vier Bände. Exemplarisch sei auf Omar Nasims Beiträge (in Band 1 und 3) zur Erforschung stellarer Nebel sowie auf Barbara Wittmanns faszinierende Studie zur zeichnerischen Bestimmung des Typusexemplars eines Fisches verwiesen, die paradigmatisch zeigen, wie Beobachten und (gleichzeitiges) Zeichnen interagieren, sich überlagern und schließlich $\mathrm{zu}$ Bildern führen, die aus ebendieser Überlagerung und nicht etwa aus ,reiner' Betrachtung hervorgegangen sind. Dass die hier versammelten Studien und 
ihre Ergebnisse vor dem Hintergrund einer Vielzahl bereits vorliegender Untersuchungen entstanden sind, liegt auf der Hand. Bruno Latours „Drawing Things Together" ist eine dieser zentralen Referenzen, doch machen Beiträge wie die beiden letztgenannten deutlich, dass es im Anschluss an Latours Erkennen der epistemischen Funktion von paperwork und des Umgangs mit Inskriptionen als immutable mobiles ebenso relevant ist, den Prozess der Hervorbringung dieser graphischen Artefakte so genau wie möglich zu rekonstruieren. Nur so wird die konstitutive Dimension des (Auf-)Zeichnens deutlich, die bei Latour wenig Beachtung findet. Selbst eine neuere, umfassende Untersuchung von Lorraine Daston und Peter Galison zur Geschichte des Objektivitätskonzepts (Daston/Galison 2010), würde von der hier umgesetzten Konzentration auf Verfahrensweisen der Sichtbarmachung profitieren, um spezielleren Fragen wie der des „scientific self“ (ebd.: 35-37) nachzugehen, aber auch der allgemeineren Frage nach dem Entstehen für objektiv befundener Bilder.

An Aspekten wie diesen wird die Anschlussfähigkeit zur aktuellen Forschung deutlich. In Bezug auf die heterogenen Zustände von Wissen, die in den hier untersuchten Verfahren entworfen werden, sind es Untersuchungsergebnisse wie das Auffinden eines „Format[s] für das akut Vorläufige und Nicht-Definitive des Denkens“ (3: 90) in den Cahiers von Paul Valéry oder eines zeichnerischen Verfahrens, "das eine Imagination kontrolliert" (3: 124), die nachhallen und gewiss großes Potential für die Beschäftigung mit vergleichbaren Materialien bergen. Gleiches gilt für die Betrachtung von Zeichnungen De Koonings, an denen Richard Shiff zeigt, wie in den gestischen Zeichenbewegungen eines sich bewegenden Körpers eine schwer zu kategorisierende Form von Körperwissen (auf-)gezeichnet und somit rezipierbar wird.

Eine der vielen Fragen, über die sich auf der Basis der hier versammelten Beiträge vortrefflich nachdenken lässt, ist also die nach den grundlegenden Differenzen wissenschaftlicher und künstlerischer Praktiken. Neben den oben erwähnten prononcierten Aufzeichnungsverfahren von Körperlichkeit scheinen es weitere Formen von Selbstreferentialität $\mathrm{zu}$ sein, die eine Unterscheidbarkeit wissenschaftlicher und künstlerischer Zeichen- und Schreibprozesse anhand dieses Kriteriums nahelegen. In diesem Sinne führt Rüdiger Campe so beeindruckend wie überzeugend vor, wie Lichtenberg ein „Verfahren Sudelbuchschreiben“ (3: 85) entwickelt und schließlich „im Sudelbuch zum Autor des Schreibens" wird (3: 83). Es scheint mir künstlerischen Arbeiten vorbehalten zu sein, Gegenstand und Verfahren so weitgehend korrespondieren zu lassen, was meines Wissens in den gewöhnlich von Wissenschaftlern durchgeführten Arbeiten nicht zu finden ist. Infolge des Verzichts auf synthetisierende Beiträge und der fehlenden Bezugnahme der Autorinnen und Autoren auf die virulente Diskussion um die Beziehung von Kunst und Wissenschaft, aktuell etwa unter dem Stichwort artistic research, ist 
die Leserin und der Leser gefordert, dies selbst zu leisten. Das ist insofern bedauerlich als gerade das Zeichnen, das in beiden Bereichen eine so zentrale Rolle spielt, einen guten Ausgangspunkt für Überlegungen zu dieser Frage bietet, wie unter anderem Dieter Mersch $(2009,2012)$ gezeigt hat.

Die Reihe Wissen im Entwurf nimmt dank ihrer weiten disziplinären Auffächerung und der konsequenten Fokussierung auf Zeichnen und Schreiben als Verfahren eine zentrale Stellung im Reigen der in jüngerer Zeit zu den diversen angrenzenden Gebieten erschienenen Publikationen ein. Dies belegt auch die Anschlussfähigkeit der Beiträge an viele der verwandten Forschungszweige, die in den letzten Jahren eine wahre Schwemme an Publikationen und Forschungsprojekten hervorgebracht haben, sei es die Forschung zu Diagrammatik, Verkörperung, extended mind, bildnerischem Denken, Schriftbildlichkeit, Notation oder auch zu etwas älteren Ansätzen wie der critique génétique. Ein Großteil der in den vier Bänden untersuchten Materialien wäre auch aus der Perspektive der genannten Forschungen produktiv zu analysieren, was die Dialogfähigkeit der hier versammelten Erkenntnisse unterstreicht.

Zugleich scheint die hier vorgenommene Fokussierung ein Forschungsgebiet des Prozeduralen in den Wissenschaften und Künsten zu umreißen, das zuvor in dieser Form nicht erkennbar war. Schließlich fügt Wissen im Entwurf dem Konzept der Papierwissenschaften durch den Verweis auf die ubiquitäre Bedeutung von Verfahren eine entscheidende Dimension hinzu. Dies bietet die Möglichkeit, die Art und Weise spezifischer zu bestimmen, in der Zeichnen und Schreiben jeweils zum Einsatz kommen, wodurch zugleich Ergebnisse zur systematisierenden Weiterverarbeitung geborgen werden. So liefert das wissenschaftliche wie das editorische Projekt durch die Verschiebung des Erkenntnisinteresses vom Prozeduralen auf das Verfahren(smäßige) sowie der methodischen Umsetzung dieser Verschiebung in Form der versammelten Studien einen Beitrag, der für die Erforschung von Wissensbildung höchst relevant ist.

\section{Anmerkungen}

1 Zu bedenken ist zudem die Möglichkeit, den Titel des Projekts wörtlich zu verstehen, was bedeuten würde, dass Wissen im Entwurf liegt und damit ein Wissensbegriff intendiert wird, der sich einer herkömmlichen „teleologischen Logik von Entwurf und Ausführung“ (1: 152) nicht mehr zuordnen ließe, sondern Wissen als (nur noch) prozessual gegeben ansieht. Entscheidender ist jedoch der Wissensbegriff, der den Praktiken der untersuchten Künstler und Wissenschaftler zugrundeliegt. In Bezug auf die Generierung und das Verständnis von Wissen ist das Anliegen eines im 19. Jahrhundert über Velazquez forschenden Kunsthistorikers offenkundig ein anderes als das eines Selbstaufzeichnungen produzierenden Künstlers ein Jahrhundert später.

2 Diese Liste ist nicht ganz rein, da sie teilweise auf Untersuchungsergebnissen der entsprechenden Beiträge, beispielsweise der Interpretation von künstlerischen Werken, 
basiert (wie die Interpretation von Kippenbergers Hotelzeichnungen als Selbstaufzeichnung).

3 Genauer: Zeichnen als Diagnose- und Therapieinstrument; untersucht wird streng genommen das Zeichnen als Verfahren der Kinder, intepretierbares graphisches Material für die Analytikerin zu generieren.

\section{Literatur}

Daston, Lorraine/Galison, Peter, 2010. Objectivity, New York: Zone Books.

Latour, Bruno, 1990: ,Drawing Things Together'.In: Michael Lynch und Steve Woolgar, Hg., Representation in Scientific Practice, Cambridge/Mass.: MIT Press, 19-68.

Mersch, Dieter, 2009. Kunst als epistemische Praxis. In: Elke Bippus, Hg., Kunst des Forschens. Praxis eines ästhetischen Denkens. Zürich, Berlin: diaphanes, 27-47.

Mersch, Dieter, 2012. Schrift/Bild - Zeichnung/Graph - Linie/Markierung. Bildepisteme und Strukturen des ikonischen >Als`. In: Sybille Krämer, Eva Cancik-Kirschbaum und Rainer Totzke, Hg., Schriftbildlichkeit. Wahrnehmbarkeit, Materialität und Operativität von Notationen. Berlin: Akademie Verlag, 305-327.

Benjamin Meyer-Krahmer

Hochschule für Grafik und Buchkunst Leipzig

Wächterstr. 11

04107 Leipzig

Deutschland

E-Mail: bmk@hgb-leipzig.de 